\title{
Neural Basis of Visually Guided Head Movements Studied With fMRI
}

\author{
Laurent Petit ${ }^{1}$ and Michael S. Beauchamp ${ }^{2}$ \\ ${ }^{1}$ Groupe d'Imagerie Neurofonctionnelle, Unité Mixte de Recherche6095, Centre National de la Recherche Scientifique-Commissariat à la \\ Énergie Atomique-Université de Caen et Université Paris 5, Centre Cyceron, Caen, France; and ${ }^{2}$ Laboratory of Brain and Cognition, \\ National Institute of Mental Health, Bethesda, Maryland 20892
}

Submitted 31 October 2002; accepted in final form 17 January 2003

Petit, Laurent, and Michael S. Beauchamp. Neural basis of visually guided head movements studied with fMRI. J Neurophysiol 89: 2516-2527, 2003. First published January 24, 2003; 10.1152/jn.00988.2002. We used event-related fMRI to measure brain activity while subjects performed saccadic eye, head, and gaze movements to visually presented targets. Two distinct patterns of response were observed. One set of areas was equally active during eye, head, and gaze movements and consisted of the superior and inferior subdivisions of the frontal eye fields, the supplementary eye field, the intraparietal sulcus, the precuneus, area MT in the lateral occipital sulcus and subcortically in basal ganglia, thalamus, and the superior colliculus. These areas have been previously observed in functional imaging studies of human eye movements, suggesting that a common set of brain areas subserves both oculomotor and head movement control in humans, consistent with data from single-unit recording and microstimulation studies in nonhuman primates that have described overlapping eye- and head-movement representations in oculomotor control areas. A second set of areas was active during head and gaze movements but not during eye movements. This set of areas included the posterior part of the planum temporale and the cortex at the temporoparietal junction, known as the parieto-insular vestibular cortex (PIVC). Activity in PIVC has been observed during imaging studies of invasive vestibular stimulation, and we confirm its role in processing the vestibular cues accompanying natural head movements. Our findings demonstrate that fMRI can be used to study the neural basis of head movements and show that areas that control eye movements also control head movements. In addition, we provide the first evidence for brain activity associated with vestibular input produced by natural head movements as opposed to invasive caloric or galvanic vestibular stimulation.

\section{N T R O D U C T I O N}

Studies of eye-head coordination suggest that oculomotor areas may control head movements as well as eye movements (reviewed in Sparks et al. 2001). Considerable information exists about the functional anatomy of eye movements in healthy humans as revealed by positron emission tomography (PET) (Anderson et al. 1994; Dorrichi et al. 1997; Fox et al. 1985a,b; Lang et al. 1994; Law et al. 1997, 1998; Nakashima et al. 1994; O'Driscoll et al. 1995; O'Sullivan et al. 1995; Paus et al. 1993; Petit et al. 1993, 1996; Sweeney et al. 1996) and blood oxygenation level dependent (BOLD) functional magnetic resonance imaging (fMRI) (Beauchamp et al. 2001; Berman et al. 1999; Bodis-Wollner et al. 1997; Corbetta et al. 1998; Darby et al. 1996; Heide et al. 2001; Luna et al. 1998;

Address for reprint requests: M. S. Beauchamp, NIMH/LBC, 10 Center Drive MSC 1366, Building 10, Room 4C104, Bethesda, MD 20892-1366 (E-mail: mbeauchamp@nih.gov).
Müri et al. 1996; Nobre et al. 2000; Petit and Haxby 1999; Petit et al. 1997). Performing eye movements leads to BOLD signal increases in a cortical network consisting of areas in the precentral sulcus (frontal eye fields, FEF), in the medial superior frontal cortex (supplementary eye fields, SEF), in the intraparietal sulcus (parietal eye fields, $\mathrm{PEF}$ ), in the precuneus, at the junction of occipital and temporal cortex (MT/V5) as well as subcortical areas including basal ganglia, thalamus, and cerebellum.

Much less is known about the functional anatomy that underlies head movement. This is largely due to the requirement that the head of the subject remains motionless during PET and fMRI scans. A recently developed technique for fMRI studies of tasks requiring brief movements (Birn et al. 1999) prompted us to examine the possibility of studying visually guided head movements using fMRI.

A fundamental difference between eye and head movements is that cranial translation and rotation stimulates the vestibular system, which serves as an important feedback mechanism for monitoring head position in space. In addition to posture and balance, this independent head-position indicator helps maintain a given direction of gaze while the head is moving (Brandt and Dieterich 1999). Functional neuroimaging studies of cortical vestibular processing have been hindered by the requirement for a motionless head during PET and fMRI scans. To provide vestibular stimulation, invasive techniques have been used, including irrigation of the external ear with cold water, (known as caloric vestibular stimulation) and mechanical vibration of the bony mastoid (known as galvanic vestibular stimulation). Invasive vestibular stimulation has shown activations of a perisylvian core region within the vestibular system, namely the parieto-insular vestibular cortex (PIVC), as well as in the temporoparietal cortex, basal ganglia, thalamus, and anterior cingulate cortex.

In our study, we first sought to discover the network of brain areas that are active when humans make visually guided head movements. Next, we wished to examine the relationship of these head movement areas to previously described oculomotor control areas. In addition, we wished to learn if head movements activate brain areas important for vestibular processing, in particular PIVC. Finally, we wished to determine if the vestibular activations produced by natural head movements

\footnotetext{
The costs of publication of this article were defrayed in part by the payment of page charges. The article must therefore be hereby marked "advertisement" in accordance with 18 U.S.C. Section 1734 solely to indicate this fact.
} 
differ from those reported for caloric irrigation and galvanic stimulation.

\section{METHODS}

\section{Subjects}

Six healthy right-handed subjects $(S 1-S 6)$, three females and three males (average age: $30.3 \mathrm{yr}$ ) underwent a complete physical examination and provided informed consent (World Medical Association 1997). Subjects were free of neurological or psychiatric illness and were compensated for participation in the study in accordance with the National Institute of Mental Health Institutional Review Board. Four additional subjects underwent psychophysical testing (1 subject participated in both psychophysical and MR testing).

\section{Visual stimulus}

The visual stimulus consisted of a central fixation cross $\left(0.7^{\circ}\right.$ wide $)$ that was always present and a small round target $\left(0.4^{\circ}\right.$ diam $)$ that was only present during movement epochs. A graphics board (Cambridge
Research Systems, Cambridge, UK) was programmed to back-project the stimulus onto a Lucite tangent screen with a video projector (Sharp USA, Montclair, NJ). The tangent screen was $98 \mathrm{~cm}$ wide at a viewing distance of $\sim 181 \mathrm{~cm}$, for a maximum visual angle of $28^{\circ}$. Targets were presented in one of eight locations evenly spaced along the horizontal meridian, which spanned the entire tangent screen (4 in each hemifield; see Fig. 1 for illustration). Stimuli appeared in alternating hemifields to maximize the amplitude of eye, head, and gaze shifts and to equate the number of left and right movements in each scan series. Within a hemifield, the target position for each stimulus presentation was randomly chosen from one of the four possible locations $\left(3.5,7,10.5\right.$, and $\left.14^{\circ}\right)$. Subjects made movements to each target as it appeared, with maximum movement amplitude of $28^{\circ}\left(14^{\circ}\right.$ $\mathrm{L}$ or $\mathrm{R}$ to $14^{\circ} \mathrm{R}$ or $\mathrm{L}$ ).

\section{Types of movements}

Subjects performed one of three different types of saccadic movement in response to the visually presented targets: eye movements to each target location with head stationary, head movements toward the
A 1. Eye movement task

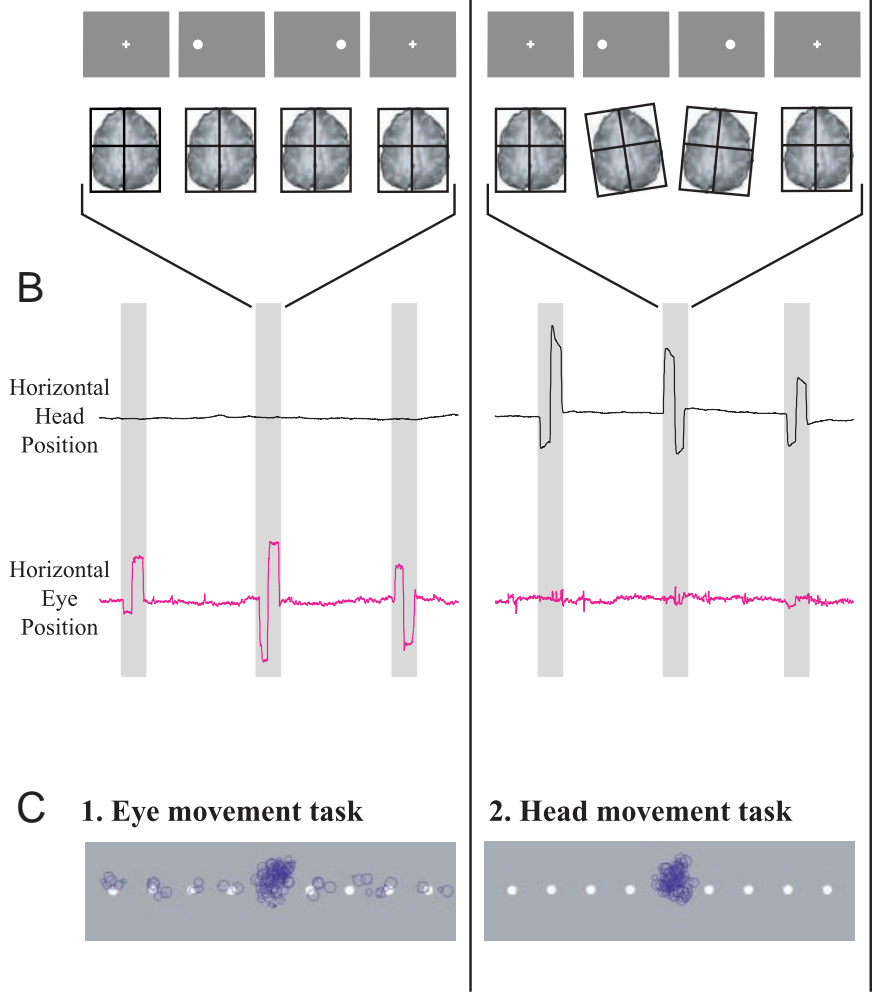

3. Gaze movement task

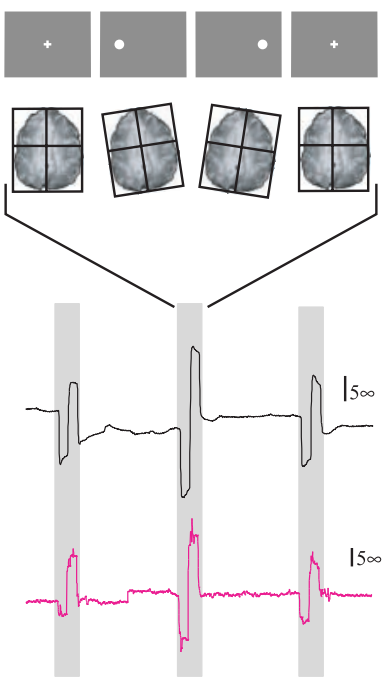

3. Gaze movement task

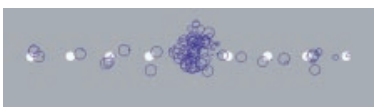

FIG. 1. Three varieties of visually guided movements and behavioral data. A: subjects viewed a target that appeared at different locations along the horizontal meridian or at fixation crosshairs. Sequential visual stimuli are shown as gray rectangles. Subjects performed 1 of 3 tasks, shown in 3 columns. Left: eye movement toward target. Middle: head movement toward target (eyes fixated centrally). Right: combined head and eye (gaze) movement toward target. Brain images were collected during task performance, allowing verification that head movements were performed during headand gaze-movement conditions but not eyemovement conditions (black grid overlaid on brain illustrates motion; for quantification, see Fig. 2A). B: head and eye movements were monitored in a separate experimental session outside the scanner. Head position trace (black line) shows angle of horizontal rotation of the head (pitch angle). Eye position trace (red line) shows horizontal eye position. Sample traces demonstrate that subjects made head movements only during head- and gaze-movement tasks and that subjects made eye movements only during eye- and gaze-movement tasks. $C$ : summary of eye-position data for an entire experimental session for 1 subject. White circles illustrate possible target locations (only 1 target was presented at a time); fixation crosshairs is located at center of display. Blue circles illustrate fixation position. For eye- and gaze-movements conditions, eyes moved to target positions when not fixating centrally. For head-movement condition, central fixation was sustained. $D$ : summary of eye-position data across subjects (error bars represent SE) shown as percentage of time spent in central fixation. Significantly more time was spent in central fixation during headmovement condition, showing that subjects were able to suppress eye movements toward targets. 
target while fixating centrally, and gaze movements (combined eye and head movements) toward the target. Subjects' heads were unrestrained inside the MR head coil, and they were able to make the required in-plane head movements without difficulty by simply turning their head to the left or right.

\section{Head- and eye-movement measurement}

Commercially available eye-tracking systems are able to monitor eye movements in the MR scanner. However, these systems require that the head be stationary and are not capable of measuring head movements or combined eye and head movements. Therefore we measured eye and head movements outside the scanner using a video tracking system (Applied Science Laboratories, Bedford, MA).

\section{MR data acquisition}

Within each 18-s trial, subjects made brief saccadic movements for $3 \mathrm{~s}$ followed by a 15 -s period in which they fixated centrally with their head still. In each 3-s movement epoch, three movements were made: a target appeared along the horizontal meridian and subjects moved toward it (1st movement); after $1 \mathrm{~s}$, the target disappeared and reappeared in a new location along the horizontal meridian and subjects moved toward it (2nd movement); after another 1-s delay, the target disappeared and subjects fixated centrally (3rd movement).
Each MR scan series contained 12 18-s trials. Before each scan series, subjects were cued by the experimenter to perform one of the three different types of movement (eye, head, or gaze). The scan series order was randomized within each subject's experimental session. Subjects were instructed to return their head to the same position (nose facing up) following each movement epoch. Subjects performed 24 trials of each movement type (divided into 2 MR scan series) with three movements in each trial, for a total of 72 movements of each type per subject.

\section{Techniques for accounting for head motion in fMRI}

Because large voluntary head movements produce gross MRI artifacts that last for the duration of the movement, head movements interfere with fMRI. We use a method first described and validated by Birn et al. (1999) to overcome this obstacle (illustrated in Fig. 2). The method uses a slow event-related design in which subjects make a brief movement $(0-5 \mathrm{~s})$ followed by a long period of inactivity $(\sim 15$ s). Blood oxygenation-level dependent (BOLD) fMRI measures hemodynamic changes that reach peak amplitude $\sim 6-10 \mathrm{~s}$ after neuronal activity and returns to baseline within $15 \mathrm{~s}$. Images collected during the movement $(0-5 \mathrm{~s})$ are contaminated by movement and are not used in the analysis. Images collected at $6-10 \mathrm{~s}$ (when the subject is stationary) are not contaminated by motion but reflect the underlying neural activity 6-10 s earlier i.e., when movements were being

\section{A Motion parameters during single MR scans}

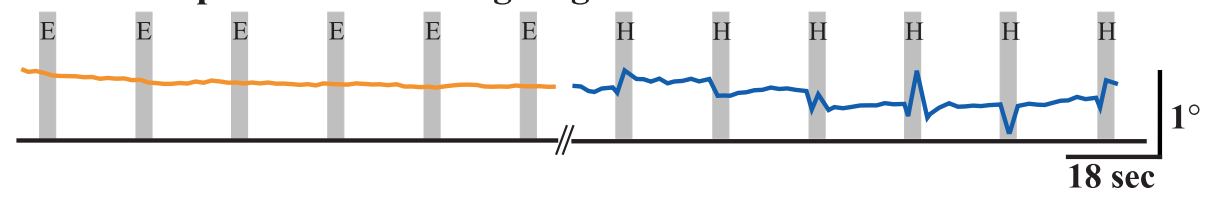

B Raw MR signal during single MR scans

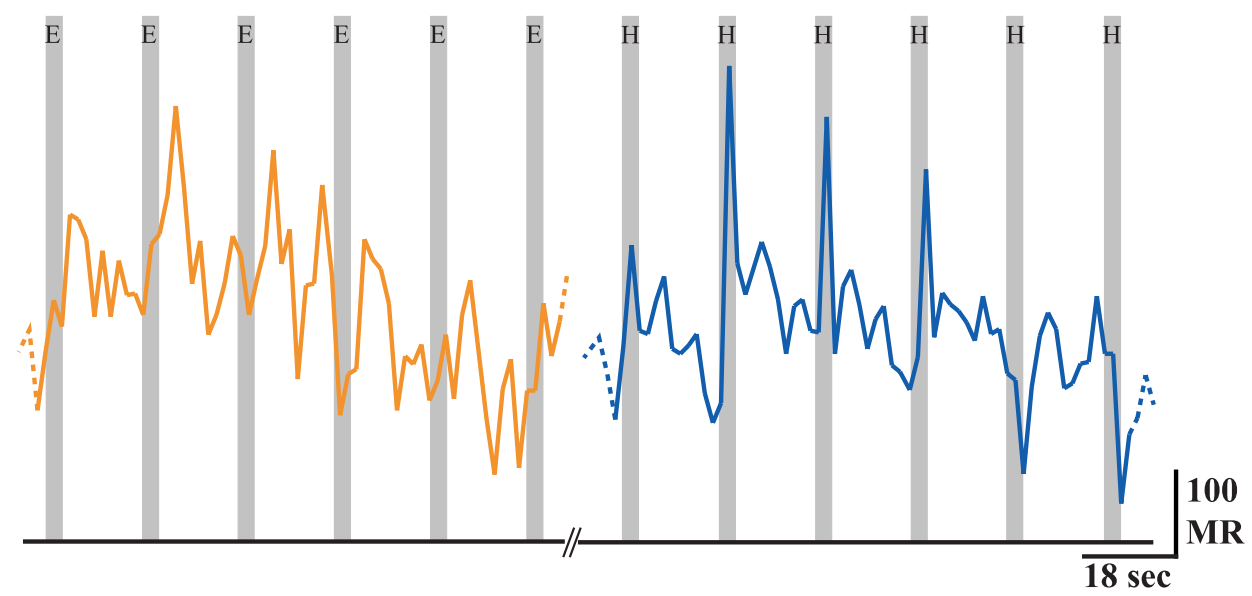

C Average MR signal during a single trial

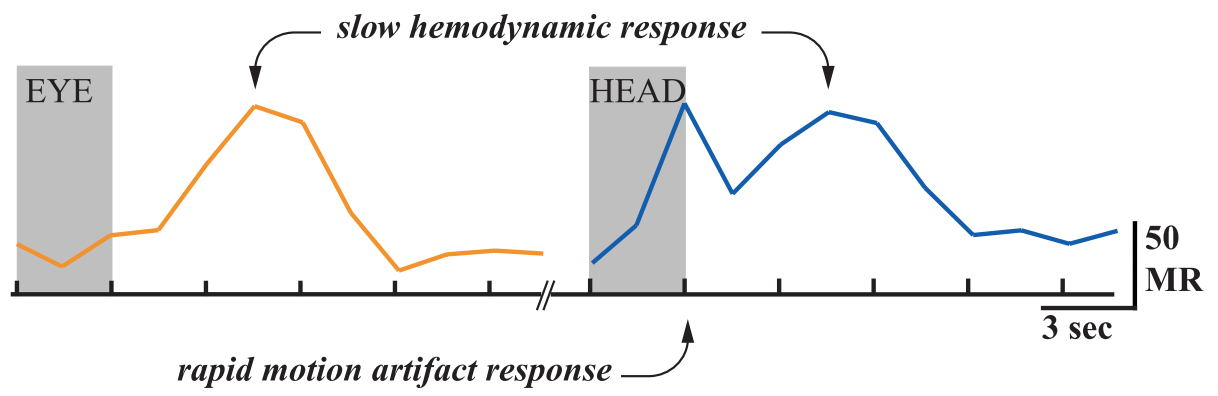

FIG. 2. Explanation of the event-related MRI methodology. Each movement trial consisted of a 3-s movement epoch (gray bars) followed by a 15 -s window in which no movements were made and the slow hemodynamic response was observed. Graphs show representative data from single subjects. A: movement parameters ( $x$-axis pitch for illustration) were estimated from the MR data using a volume registration algorithm. During the eye-movement scan series (orange line), no motion was observed during motion epochs (gray bars labeled "E") or the intervening hemodynamic response windows. During the head-movement scan series (blue line), motion was observed during motion epochs (gray bars labeled "H") but not during hemodynamic response windows. $B$ : an MR time course from a single voxel shows stability when the subject made eye movements (gray "E" bars) but not during head movements (gray "H" bars). Both types of movement types evoked a slow hemodynamic response (intervals between gray bars). $C$ : the MR time course from a single voxel averaged across all eye-movement trials and all head-movement trials. Note that the motion artifact peaks during the 3-s head-movement epoch and is not seen during the eye-movement epoch. The slow hemodynamic response evoked by both movement types peaks 6-9 s after the presentation of the first target. Vertical scale bars illustrate image intensity (in arbitrary MR units). 
made. Movements collected during this interval can therefore be used to construct activation maps that show brain areas active during head movements. In effect, the Birn method reduces the very difficult problem of fMRI of rapid voluntary movements to the much simpler problem of fMRI of stationary subjects, a problem that has been effectively addressed in hundreds of fMRI studies.

As in other fMRI studies, subjects might move gradually during the fixation epoch, or they might make sudden movements (such as a sneeze) and not return to the same position as they were in previously. To address these problems, we used well-established techniques. First, brain volumes during the fixation epoch were registered to the first volume collected after the high-resolution anatomy using a leastsquares algorithm (Cox and Jesmanowicz 1999). The motion-correction algorithm accurately measures and corrects the gradual head movements that occur, for example, when the foam padding underneath the subject's head compresses or the subject slowly slides down the scanner bed (Fig. 2A). To further minimize the effects of motion on the activation map, the motion parameters calculated by the registration algorithm were used as regressors of no interest in the regression model along with a second-order polynomial to account for slow drifts in the MR signal caused, for example, by gradient coil heating. In an additional effort to eliminate any high-spatial frequency transients associated with motion, a spatial filter of root-mean-square width of $4 \mathrm{~mm}$ was applied to the echo-planar data. The effectiveness of these methods are illustrated by the concentrated, highly significant activation foci we observed (uncorrected head movement would tend to reduce the significance of activations and smear them out over a large spatial extent). Animations of actual fMRI data showing the head motion during movement epochs and the stability of cortical landmarks during fixation epochs can be viewed at http:// jn.physiology.org/cgi/content/full/00988.2002/DC1.

\section{Auditory and somatosensory stimulation}

Our experimental hypothesis was that head movements would evoke activity in vestibular cortex. Head movements in a supine position could also evoke activity in auditory cortex if the movement of the head as it rested on the foam cushion inside the RF coil resulted in acoustic stimulation that was audible over the $90+\mathrm{dB}$ scanner noise and the earplugs worn by subjects. To disambiguate auditory and vestibular activations, we mapped auditory cortex in two subjects (results shown in Fig. 7A). Auditory cortex was mapped using an Avotec SilentScan system (Avotec, Stuart, FL) which allows hearing protection and auditory stimulus delivery with an MR-compatible headset. Auditory stimuli consisted of 21-s blocks containing seven 3 -s stimulus epochs, alternating with $21 \mathrm{~s}$ of silence (no stimulation). Three types of auditory stimuli containing natural and synthesized waveforms were used, each containing a mix of frequencies from 50 $\mathrm{Hz}$ to $15,000 \mathrm{kHz}$ to ensure that auditory cortex in its entirety was activated.

In addition to vestibular cortex, head movements in the supine position could evoke activity in somatosensory cortex as the motion of the head on the foam cushion stimulated mechanoreceptors in the skin on the back of the head and neck. To test this possibility, we studied brain responses to tactile stimulation of the head and neck in two subjects. A tank with compressed air was connected to a computercontrolled valve which delivered brief (valve open time: $100 \mathrm{~ms}$ ) air puffs at a rate of $2 \mathrm{~Hz}$. The air puffs were delivered with a plastic tube directed to the back of the head and neck at approximately the same region as would be stimulated with head movements. Eighteen seconds of stimulation alternated with $18 \mathrm{~s}$ of no stimulation. Regions responsive to somatosensory stimulation are shown in Fig. $7 B$.

\section{MRI procedures}

Subjects were examined using a 3 Tesla MR scanner (General Electric, Milwaukee, WI) with a volume transmit and receive coil that provided whole head coverage. At the beginning of each scan session, a high-resolution anatomical scan was acquired (whole-brain T1weighted spoiled-grass; $0.9375 \times 0.9375-\mathrm{mm}$ in-plane resolution, 1.2-mm slice thickness). Functional scan series were collected using a gradient-echo echo-planar sequence with a repetition time of 1,500 $\mathrm{ms}$, an echo time of $30 \mathrm{~ms}$ and in-plane resolution $3.75 \times 3.75 \mathrm{~mm}$. Twenty-four axial slices with a thickness of $5 \mathrm{~mm}$ were collected to provide coverage of the entire cortex. 150 volumes were collected in each scan series, and the first six volumes in each series (collected before equilibrium magnetization was reached) were discarded.

\section{Data analysis}

Data were visualized and analyzed using AFNI (Cox 1996; Cox and Hyde 1997). Multiple regression was used to select voxels that demonstrated a neural-hemodynamic response to the movement (6- to 10-s latency and 10- to 15-s duration) and ignore voxels that demonstrated only a motion-artifact response (0-s latency, 3-s duration). The two brain volumes collected during each 3 -s movement epoch were ignored in the analysis. These volumes were contaminated by movement during the head movement trials, and to prevent statistical bias, the corresponding data from eye-movement trials was also ignored (although it was not contaminated by movement; see also Techniques for accounting for head motion in $\mathrm{fMRI}$ ).

Figure 2 illustrates an MR time course for a single trial of eye- and head-movement tasks. Regressors of interest were created to fit the expected long-latency, long-duration hemodynamic response using a gamma variate function $t^{b} e^{-t / c}$, with $b=8.6, c=0.547$ (Cohen 1997). Three separate regressors of interest were used to independently model the activation due to each type of motion (eye, head, and gaze). Voxels were considered active if the total variance accounted for by the best-fit combination of the regressors of interest exceeded a conservatively chosen $F$ ratio of $9\left(P<10^{-5}\right.$ per voxel, uncorrected for multiple comparisons). This analysis detected brain regions active during any combination of movement type. After active voxels were detected, the responses within these voxels to different movement conditions were compared with a more liberal threshold $(P<0.05)$ to search for subtle differences between conditions. Active voxels with $P<0.05$ for the contrast of eye movements versus head movements were considered head movement specific (blue color scale in figures), whereas voxels with $P>0.05$ were considered nonspecific (orange color scale in figures). Active voxels were interpolated to $1 \mathrm{~mm}^{3}$ using a cubic interpolation algorithm and overlaid on each subject's highresolution anatomical scan before conversion to the standardized space of (Talairach and Tournoux 1988).

To examine the actual response to each type of movement (free of any assumptions about the shape of the response or the relative amplitude of the response across conditions), average MR time series were created from anatomically defined regions in each subject. An average MR time series was created from all active voxels in each region in each subject, and a mixed-effects model (with subject as the random factor) was used to perform statistics on the grand mean time series. To create the average activation maps shown in the figures, a fixed-effects approach was used (activation amplitude and $t$-values were averaged in each voxel in standardized space across subjects). Similar results were obtained using a random-effects analysis in which an ANOVA was performed on the amplitude of activation in each condition in each voxel across subjects (statistical values from the ANOVA are shown in Tables 1 and 2).

\section{Volumes of interest (VOIs)}

Examination of the individual subject activation maps and the group activation map revealed activity in a distributed cortical and subcortical network of brain areas during visually guided saccadic movements. To compare the response of these different regions to eye, head, and gaze movements, the MR response to each movement 
TABLE 1. Brain areas active during visually guided movements (eye, head, or gaze), averaged across six subjects

\begin{tabular}{|c|c|c|c|c|c|c|}
\hline \multirow[b]{2}{*}{ Volumes of Interest } & \multirow[b]{2}{*}{$t$-Score } & & \multicolumn{3}{|c|}{ Coordinates } & \multirow{2}{*}{$\begin{array}{l}\text { Volume } \\
\mathrm{mm}^{3}\end{array}$} \\
\hline & & & $x$ & $y$ & $z$ & \\
\hline \multirow[t]{2}{*}{ Precentral sulcus (superior) } & 27.7 & $\mathrm{~L}$ & -26 & -14 & 53 & 5443 \\
\hline & 32.7 & $\mathrm{R}$ & 31 & -8 & 52 & 5818 \\
\hline \multirow[t]{2}{*}{ Precentral sulcus (inferior) } & 21.6 & $\mathrm{~L}$ & -44 & -14 & 40 & 3337 \\
\hline & 20.7 & $\mathrm{R}$ & 47 & -6 & 40 & 4625 \\
\hline Medial superior frontal gyrus & 32.7 & & 0 & -6 & 54 & 10891 \\
\hline \multirow[t]{2}{*}{ Intraparietal sulcus } & 20.0 & $\mathrm{~L}$ & -14 & -74 & 43 & 5600 \\
\hline & 18.6 & $\mathrm{R}$ & 22 & -54 & 44 & 8502 \\
\hline Precuneus & 15.3 & & 14 & -42 & 47 & 4141 \\
\hline \multirow[t]{2}{*}{ Lateral occipital sulcus } & 23.2 & $\mathrm{~L}$ & -55 & -59 & 2 & 4099 \\
\hline & 28.8 & $\mathrm{R}$ & 58 & -51 & 12 & 4907 \\
\hline \multirow[t]{2}{*}{ Caudate nucleus } & 26.1 & $\mathrm{~L}$ & -12 & 6 & 12 & 3839 \\
\hline & 16.3 & $\mathrm{R}$ & 15 & 3 & 16 & 975 \\
\hline \multirow[t]{2}{*}{ Lenticular nucleus } & 13.5 & $\mathrm{~L}$ & -22 & -1 & 8 & 3089 \\
\hline & 12.4 & $\mathrm{R}$ & 24 & -3 & 8 & 3245 \\
\hline \multirow[t]{2}{*}{ Thalamus } & 20.4 & $\mathrm{~L}$ & -11 & -16 & 8 & 5641 \\
\hline & 20.3 & $\mathrm{R}$ & 9 & -16 & 8 & 3866 \\
\hline \multirow[t]{2}{*}{ Superior colliculus } & 13.3 & $\mathrm{~L}$ & -7 & -26 & -1 & 79 \\
\hline & 17.8 & $\mathrm{R}$ & 8 & -26 & -1 & 79 \\
\hline
\end{tabular}

The first column is the location of anatomically defined volumes of interest (VOIs). The second column shows the peak $t$-statistic within each active region for the contrast movement versus rest obtained from an across-subjects voxelwise ANOVA with 10 degrees of freedom (note that 32.7 represents the maximum calculable $t$-value). The third column lists the hemisphere (L, left; R, right) for VOIs on the lateral surface of the hemisphere and the subcortical VOIs. Activity in both hemisphere was grouped for medial VOIs. The fourthsixth columns are $(x, y, z)$ normalized Talairach coordinates of the center of mass of the activity in each VOI in millimeters from the anterior commissure. The seventh column lists the volume of cortex in each VOI that showed an overall experimental effect (visually guided saccadic movements vs. fixation). Data correspond to Fig. $3 B$.

type was averaged across the active voxels in each region in each subject. Then the response within each region was averaged across subjects. This analysis method allowed close inspection of the actual response to each movement type in different regions, while allowing for anatomical variability across subjects.

Anatomical landmarks for cortical and subcortical oculomotor control areas are well established (Beauchamp et al. 2001; Petit and Haxby 1999; Petit et al. 1993). For oculomotor control areas, 20 volumes of interest were manually traced on each subject's highresolution anatomical scan using that subject's anatomical landmarks without reference to the functional data. Because relatively little is known about cortical areas important for head movements and vestibular processing, for these areas we used a post hoc approach of identifying areas that were consistently active during head movements (through examination of the group activation map) and then creating VOIs for these areas in each individual subject. In all cases, volumes of interest were not drawn on a single slice but were traced on multiple slices through the volume.

At the cortical level, two VOIs delineated bilateral precentral regions cortex encompassing the precentral gyrus and the precentral sulcus (PreCS), including $5 \mathrm{~mm}$ on the anterior bank of the sulcus from the junction with the superior frontal sulcus to the lateral convexity. The VOI for inferior precentral cortex extended along the PreCS from $20 \mathrm{~mm}$ above the bicommissural plane (AC-PC) to 10 $\mathrm{mm}$ above the intersection of the PreCS with the inferior frontal sulcus. The VOI for superior precentral cortex extended superiorly along the remainder of the sulcus. The VOI delineating the dorsomedial part of the superior frontal gyrus consisted of $12 \mathrm{~mm}$ of cortex on each side of the interhemispheric fissure anterior to vertical plane passing through the posterior commissure (VPC) and extending forward to the anterior convexity. Its inferior limit corresponded to the cingulate sulcus in the posterior part and to the plane $45 \mathrm{~mm}$ above $\mathrm{AC}-\mathrm{PC}$ in the anterior part. This inferior limit was chosen to delineate the medial part of Brodmann area 6 that contains both the supplementary motor area (SMA) and the SEF (Petit et al. 1998; Picard and Strick 1996). The VOI delineating intraparietal sulcus (IPS) included the cortex on both banks of the sulcus, namely both superior and inferior parietal lobules, from the junction with the postcentral sulcus to the posterior convexity. Its inferior limit corresponded to the plane $30 \mathrm{~mm}$ above the AC-PC plane and thus included the deepest part of the IPS. The VOI delineating bilateral regions at the lateral junction of the temporal and occipital cortex was centered on the junction of the ascending limb of the inferior temporal sulcus and the lateral occipital sulcus (LOS). Its anterior limit corresponded to the coronal plane 40 $\mathrm{mm}$ posterior to the plane passing through the anterior commissure (VAC) and extending backward to the coronal plane $85 \mathrm{~mm}$ posterior to the VAC. Its superior limit corresponded to the plane $12 \mathrm{~mm}$ above the AC-PC plane, and its inferior limit corresponded to the plane 4 $\mathrm{mm}$ below the AC-PC plane. This region was defined to include the area that is homologous to monkey MT/MST, also called V5 (Tootell and Taylor 1995; Watson et al. 1993; Zeki et al. 1991). The VOI delineating the precuneus consisted of $15 \mathrm{~mm}$ of parietal cortex on each side of the interhemispheric fissure, posterior to the marginal ramus of the cingulate sulcus and extended backward to the posterior convexity. Its inferior limit corresponded to the plane $30 \mathrm{~mm}$ above the AC-PC plane. The two last cortical VOIs delineated the medial part of the occipital cortex including both striate and extrastriate visual areas on both sides of AC-PC.

At the subcortical level, two VOIs delineated bilateral caudate nucleus including its head and its body. Two VOIs delineated bilateral lenticular nuclei including both the putamen and the globus pallidus. Two VOIs delineated bilateral thalamus and the two last corresponded to the superior colliculus (SC). This later contained all visible SC tissue, while excluding as much of the surrounding fluid and non-SC tissue as possible.

\section{R E S U L T S}

\section{Behavioral results}

Figure 1 shows behavioral data collected inside and outside the MR scanner. Subjects were able to accurately perform each

TABLE 2. Brain areas significantly more active during head movements than eye movements, averaged across six subjects

\begin{tabular}{|c|c|c|c|c|c|c|}
\hline \multirow[b]{2}{*}{ Volumes of Interest } & \multirow[b]{2}{*}{$t$-Score } & & \multicolumn{3}{|c|}{ Coordinates } & \multirow{2}{*}{$\begin{array}{c}\text { Volume } \\
\mathrm{mm}^{3}\end{array}$} \\
\hline & & & $x$ & $y$ & $x$ & \\
\hline \multirow[t]{2}{*}{ Perisylvian region } & 4.9 & $\mathrm{~L}$ & -44 & -31 & 15 & 594 \\
\hline & 5.8 & $\mathrm{R}$ & 44 & -33 & 13 & 662 \\
\hline \multirow[t]{2}{*}{ Postcentral sulcus } & 3.1 & $\mathrm{~L}$ & -19 & -33 & 60 & 24 \\
\hline & 4.2 & $\mathrm{R}$ & 21 & -29 & 56 & 93 \\
\hline Paracentral lobule & 4.3 & & 4 & -17 & 55 & 351 \\
\hline Supramarginal gyrus & 8.3 & $\mathrm{R}$ & 47 & -21 & 23 & 127 \\
\hline Superior parietal gyrus & 3.6 & $\mathrm{R}$ & 26 & -44 & 56 & 351 \\
\hline Cuneus & 5.8 & $\mathrm{R}$ & 11 & -83 & 25 & 172 \\
\hline
\end{tabular}

The first column is the location of anatomically defined VOIs. The second column shows the peak $t$-statistic within each active region for the contrast head or gaze movements versus eye movements obtained from a voxel-wise ANOVA with 10 degrees of freedom. The third column lists the hemisphere (L, left; R, right) for VOIs on the lateral surface of the hemisphere and the subcortical VOIs. Activity in both hemisphere was grouped for medial VOIs. The fourth-sixth columns are $(x, y, z)$ normalized Talairach coordinates of the center of mass of the activity in each VOI, in mm from the anterior commissure. The seventh column lists the volume of cortex in each VOI that showed a significant effect of the contrast head or gaze movements vs. eye movements. Data correspond to Fig. $5 B$. 
of the three tasks, suppressing head movements during the eye-movement condition while tracking the target with the head during head- and gaze-movement conditions. During the head-movement condition, subjects were able to fixate centrally while moving their head toward the target (Fig. 1C).

\section{Overall eye-, head-, and gaze-movement-related activity}

Voxels exceeding the significance threshold for the main effect of interest (experimental conditions vs. central fixation) were first overlaid on each subject's anatomical images. This revealed brain areas significantly active during the three movement types (eye movements to each target location, with head stationary; head movements toward the target while fixating centrally; and combined eye and head movements toward the target, i.e., gaze movements). A similar broadly distributed set of active brain areas was observed in each subject (data from subject $S 3$ depicted in Fig. 3A; group activation map in Fig.
$3 B)$. On the lateral surface of the hemisphere, activity was observed in PreCS, IPS, and LOS. On the medial surface, activity was observed in the medial part of the superior frontal gyrus and in precuneus as well as in medial occipital cortex. At the subcortical level, activity was observed in both caudate and lenticular nucleus, the thalamus and the superior colliculus.

To allow quantitative comparisons across subjects, 20 anatomically defined VOI were manually traced in each subject. For each subject, voxels within each VOI that showed a significant response to the main effect of interest (eye, head, and gaze movements vs. central fixation) were grouped $\left(P<10^{-12}\right.$ corrected). This allowed the calculation of the volume and Talairach coordinates of activity in each VOI in each subject. Table 1 lists these values, averaged across subjects. There was no significant difference between hemispheres in volume of active regions but the IPS. In the IPS, there was $40 \%$ more active cortex in right hemisphere than in left hemisphere $(P=0.02)$.
A Representative individual subject (S3)
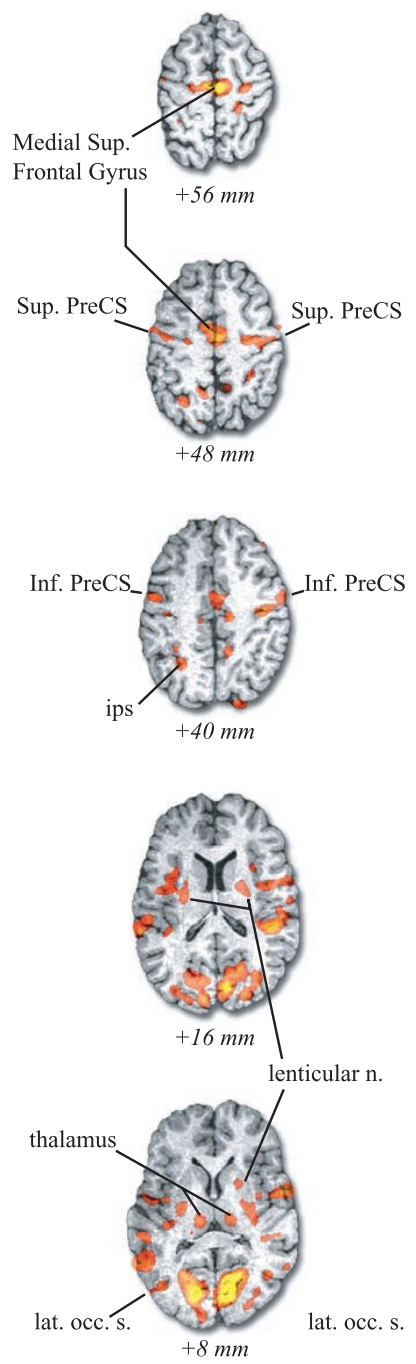

\section{B Group average data}

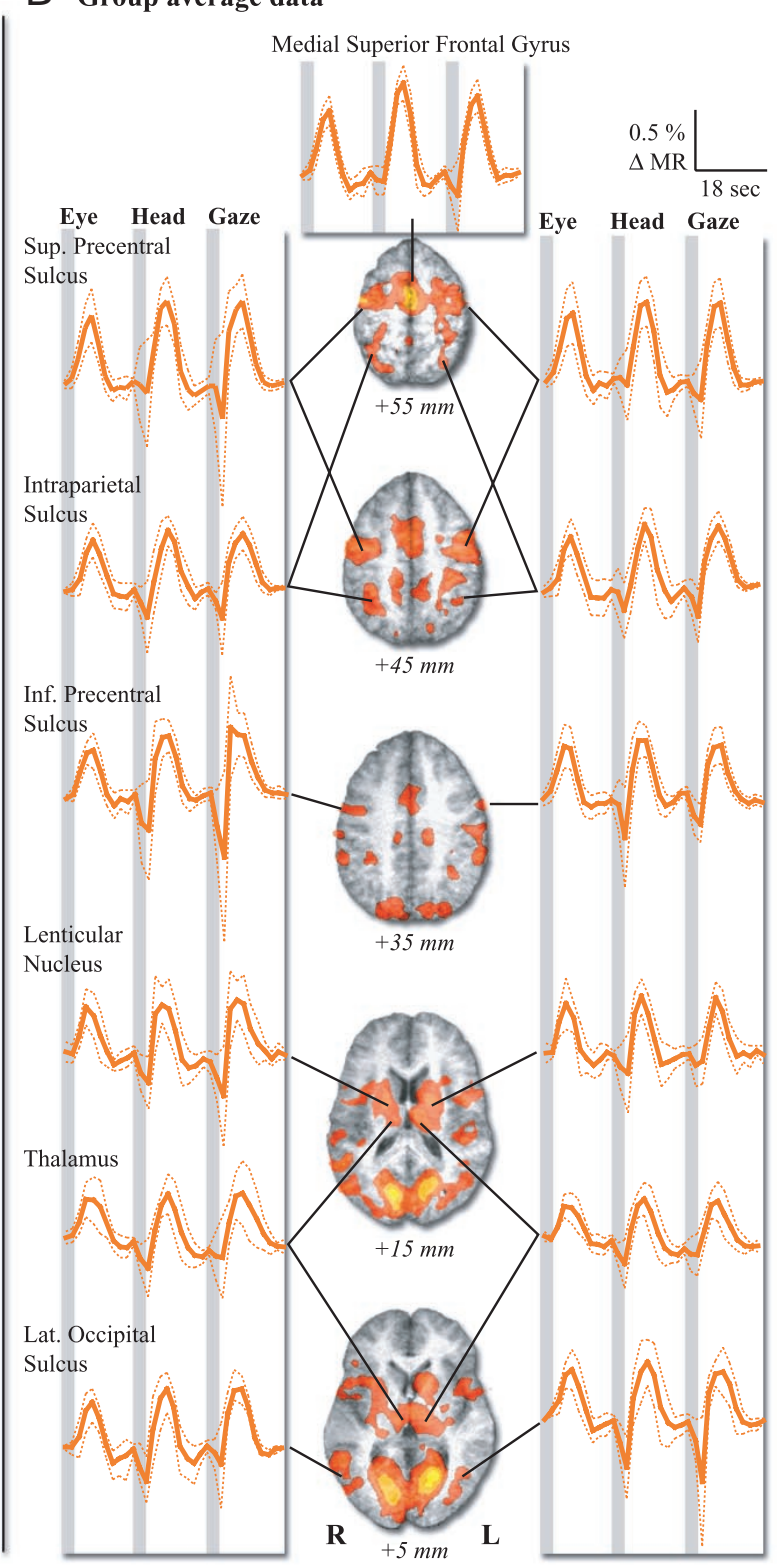

FIG. 3. A: movement related activity (colored regions) from an individual subject (S3) superimposed onto that subject's anatomical dataset (grayscale). Orange color scale shows voxels with an overall experimental effect $\left(P<10^{-5}\right)$ but showing no significant difference between eye and head movement conditions $(P>0.005)$. From top to bottom, 4 transaxial slices from superior to inferior in standard space (Talairach coordinates below each slice) with anatomical labels (sup., superior; PreCS, precentral sulcus; inf., inferior; n., nucleus; lat. occ. s., lateral occipital sulcus). $B$ : movement-related brain areas in an average activation map from 6 subjects overlaid on the average anatomical dataset from the same subjects (color scale as in A). Summary of activation in Table 1. Insets: the average MR time series from all active voxels in each volume of interest in left hemisphere (left; labeled) and right hemisphere (right). Solid graph line indicates grand mean across subjects, dashed lines indicates mean $\pm \mathrm{SE}$. Insets: each graph shows the response to 3 movement types: eye movements only; head movements only; and combined eye and head movements, respectively (labeled at top of column). Each movement trial consisted of a 3-second movement epoch (gray bars) followed by a 15-s window in which no movements were made and the slow hemodynamic response was observed. Right is left in all slices. 
A Individual subject activation in $\mathrm{SC}$
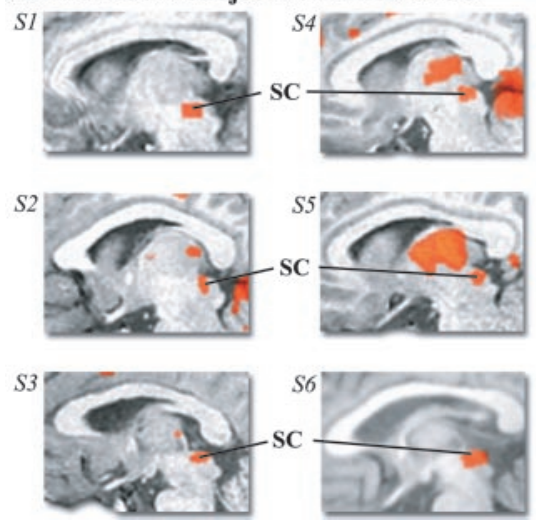

To examine the response to eye, head, or gaze movements, an average MR time series across subjects was created for each VOI for each type of saccadic movements. When subjects made saccadic eye, head, or gaze movements, an event-related MR response was observed (Fig. $3 B$ ). In each VOI, similar high-amplitude responses were observed for head movements and for gaze movements; slightly smaller responses were evoked in each VOI for eye movements alone. This effect (head/gaze $>$ eye) was largest in the medial superior frontal region (0.86 vs. $0.58 \%$, paired $t$-test, $P=0.004)$, the left superior $(0.74$ vs. $0.59 \%, P=0.04)$, and inferior $(0.61$ vs. $0.50 \%, P=0.04)$ PreCS, in the precuneus $(0.62$ vs. $0.37 \%$, $P=0.007)$ and the right $\operatorname{LOS}(0.51$ vs. $0.39 \%, P=0.04)$. The effect was smaller in the right superior $(0.81$ vs. $0.62 \%, P=$
$0.06)$ and inferior ( 0.62 vs. $0.44 \%, P=0.1)$ PreCS, bilaterally in IPS (left: $P=0.1$; right: $P=0.09)$ and in the left $\operatorname{LOS}(P=$ $0.4)$. There was no significant difference in the response amplitude for gaze movements and head movements.

Although the SC is known to play a key role in eye-head coordination, it has not been observed consistently in previous imaging studies. As shown in Fig. 4A, we observed collicular activation in each subject. In contrast to the pattern of activity observed in cortical regions (head/gaze significantly greater than eye movements), the SC showed no significant difference between the response to head/gaze movements and eye movements, and a trend was observed in the opposite direction (greater response to gaze movements).

Activity was also observed in brain stem regions inferior to

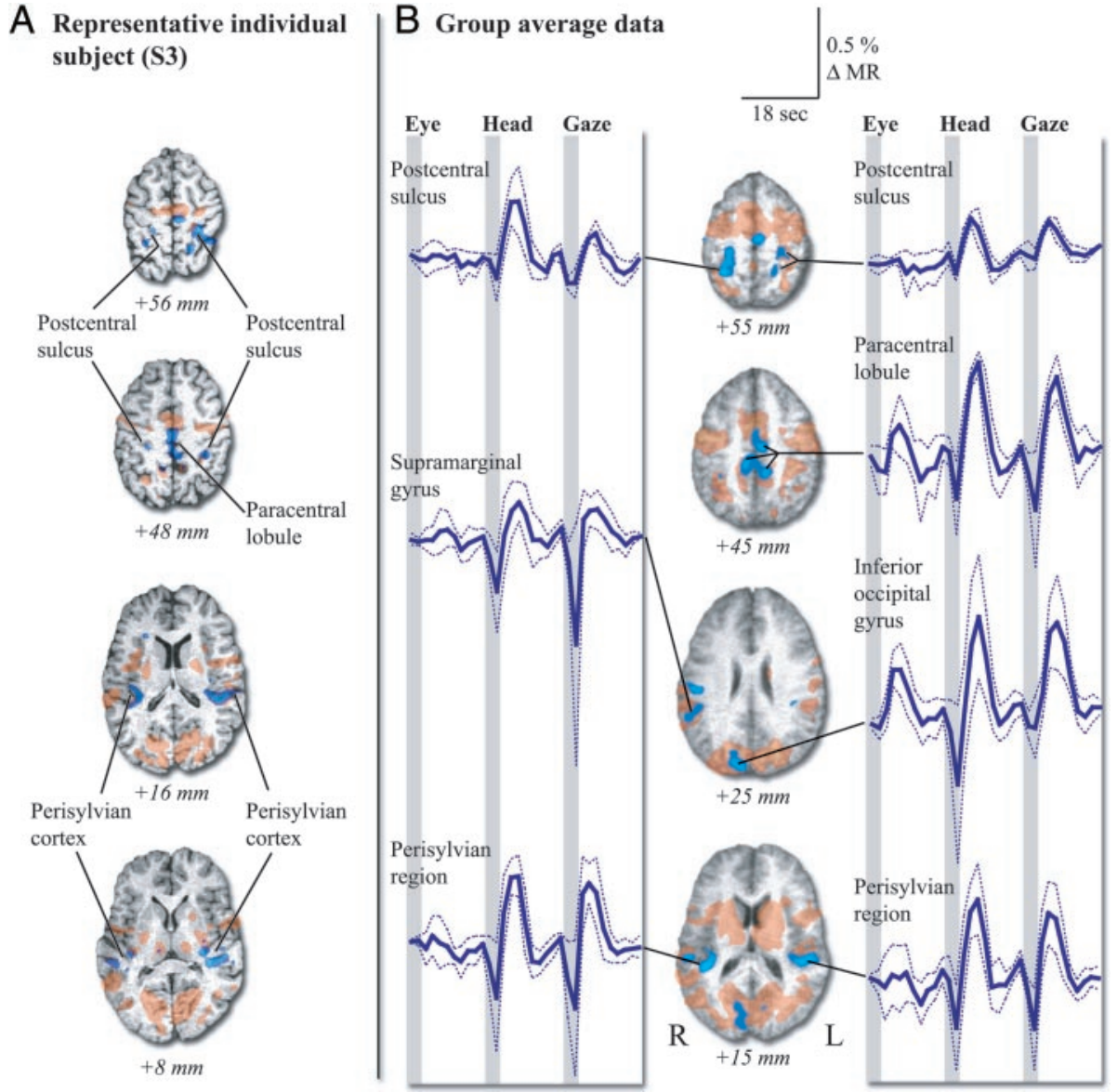

FIG. 5. A: movement-related activity (colored regions) from an individual subject $(S 2)$ superimposed onto that subject's anatomical dataset (grayscale). Blue color scale: voxels showing an overall experimental effect $\left(P<10^{-5}\right)$ and a significant preference for head movements compared with eye movements $(P<0.05)$. Orange color scale: voxels showing an overall experimental effect $\left(P<10^{-5}\right)$ but showing no significant difference between eyeand head-movement conditions $(P>0.05)$. From top to bottom, 4 transaxial slices from superior to inferior in standard space (Talairach coordinates below each slice) with anatomical labels (PostCS: postcentral sulcus). $B$ : movement-related brain areas in an average activation map from six subjects (color scale as in A). Summary of activation coordinates in Table 2. For head-movement-preferring regions (blue voxels), insets show the average MR time series from each region in left hemisphere (left) and right hemisphere (right). Inset: each graph shows the grand mean across subjects (thick central line) and the SE (thin dashed line) of the response to the 3 movement types. Right is left in all slices. 
the SC, possibly corresponding to other nuclei important in the control of eye and head movements, such as the pontine reticular formation. However, the small size and lack of anatomical distinction of these areas makes it difficult to draw conclusions about the observed activity (see DISCUSSION).

\section{Specific head- and gaze-movement-related activity}

All of the brain areas active during eye movements were also active during head and gaze movements. However, an additional set of areas that responded strongly when subjects executed head or gaze movements but showed little or no response when subject made eye movements (single subject map in Fig. $5 A$, group map in $5 B$ ).

The largest volume of head-movement-related activity was observed in the perisylvian region (volumes and coordinates of head movement related activity in Table 2). All subjects showed activation centered posterior to the Heschl's gyrus, including the posterior part of the planum temporale. As illustrated in Fig. 6 for a representative individual subject $(S 2)$, the perisylvian activation extended upward to the temporoparietal junction at the level of the parietal operculum and the bottom of the supramarginal gyrus. In addition, a distinct and more superior activation was observed in the supramarginal gyrus in all subjects in the right hemisphere (Figs. 5 and 6). Vestibular activation was located posterior, superior, and medial to auditory cortex (Fig. 7A).

Bilateral head movement related activity was also observed in the postcentral gyrus (PostCS) and on the medial surface of the hemisphere in the paracentral lobule. PostCS activation during head movements overlapped with regions responding to somatosensory stimulation of the head and neck (Fig. 7B). Right-lateralized activation was found in the supramarginal gyrus, the superior parietal gyrus, and the right cuneus. No activation was observed individually or in the group average map in the insula. No areas responded to gaze movements but not head movements or vice versa.

To better understand the response of the head-movementrelated areas, average MR time series were created for each area from voxels exceeding a significance threshold for head movements $\left(P<10^{-5}\right.$ corrected $)$ in the group activation map (Fig. 5B). All areas except the cuneus showed similar, largeamplitude responses to head and gaze movements and little or no response to eye movements. The cuneus showed a response to eye movements, but it was of smaller amplitude than the response for gaze movements $(P=0.03)$.

\section{I S C U S S I O N}

Our results demonstrate that event-related fMRI can be used to study the neural basis of visually guided head movements. Head movements are subserved by a network that shares many common areas with eye movements, including the FEFs and the SC. In addition to oculomotor control areas, head movements also activated areas important for vestibular processing, including PIVC. This PIVC activity provides the first evidence for brain activity associated with vestibular input produced by natural head movements as opposed to invasive caloric and/or galvanic vestibular stimulation.
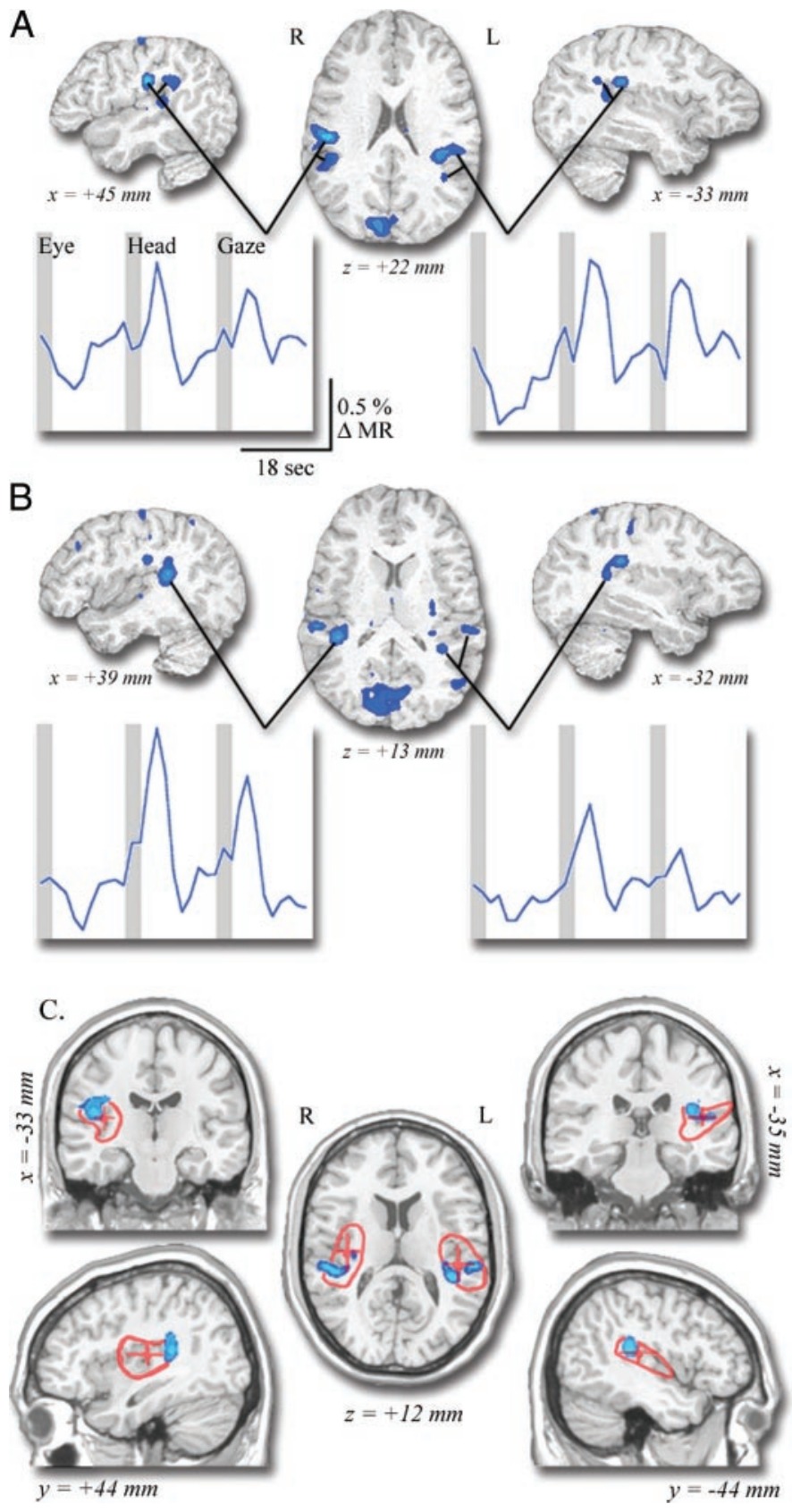

FIG. 6. Brain areas showing an overall experimental effect $\left(P<10^{-5}\right)$ with increased activity during head movements but not during eye movements $(P<$ 0.005). A: data from a single subject (S2) show a large activation in parieto-insular vestibular cortex (PIVC). Insets: average time series from active voxels within the PIVC. Also note medial occipital (cuneus) activation visible posteriorly in both axial slices (see Fig. 5 and DISCUSSION for details). B: group data showing head-movement-related activity observed around the posterior insula in the average activation map from the present study (blue voxels) and PIVC-related activations (white lines) described in previous PET and fMRI studies using invasive vestibular stimulation (Bottini et al. 1994; Dieterich et al. 1998; Lobel et al. 1998; Bense et al. 2001; Bottini et al. 2001; Deutschländer et al. 2002). Red contours show the limit of previously reported PIVC activations while the white dots and bars show the mean \pm SD coordinates [Left: $x=-44 \pm 10, y=-27 \pm 12, z=$ $12 \pm 6$; right: $x=38 \pm 6, y=-18 \pm 13, z=11 \pm 7(\mathrm{~mm})]$. All activations were superimposed on the SPM single subject MNI stereotactic brain.

\section{Overall eye-, head-, and gaze-movement-related activity}

Head and gaze movements resulted in activations in a set of regions that previous imaging studies have shown to be crucial 


\section{A Auditory Cortex vs. Vestibular Cortex}
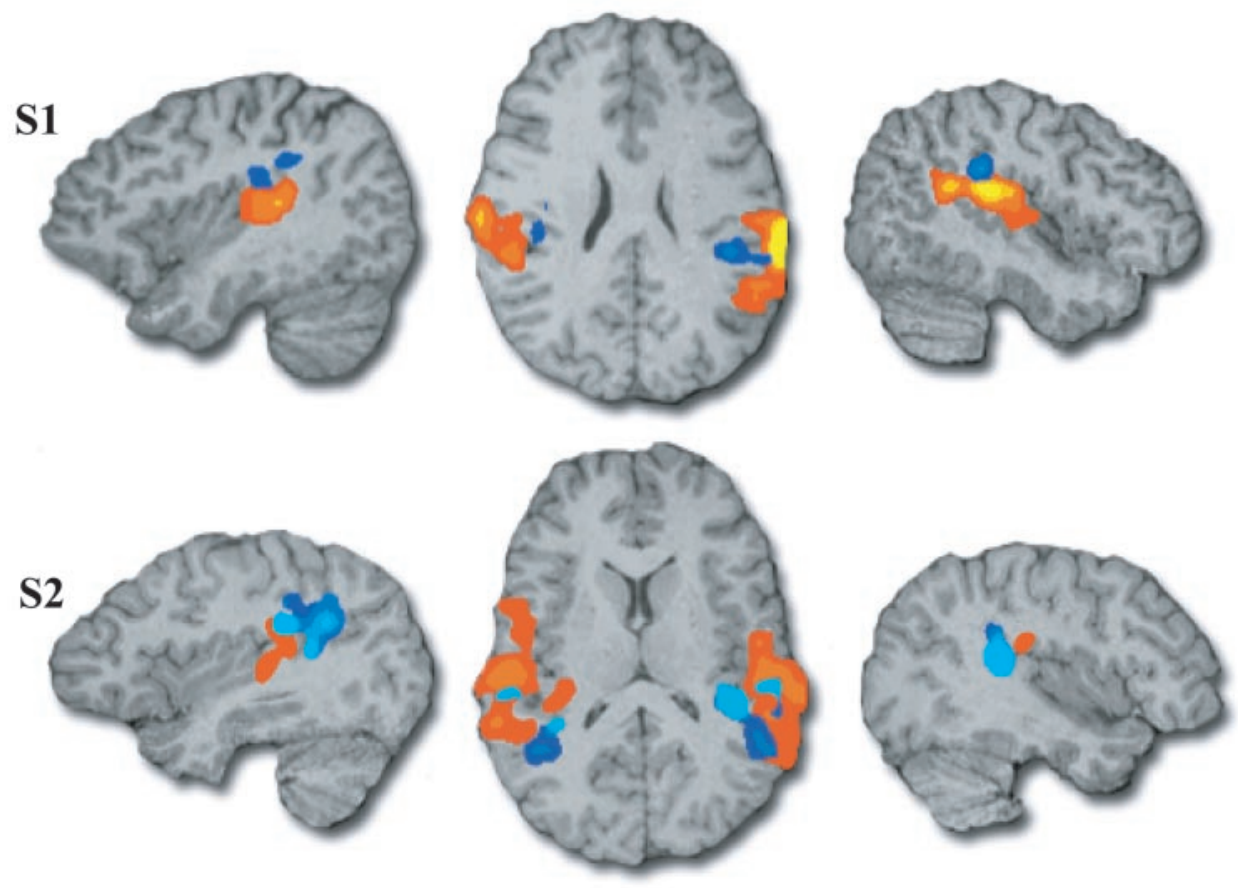

FIG. 7. Comparison between activity evoked by head movements and activity evoked by auditory and somatosensory stimulation in 2 subjects ( $S 1$ and $S 2$ ). A: blue colors illustrate regions of PIVC responding to head movements. Orange colors show regions responding to broad-spectrum auditory stimuli consisting of natural and synthesized sounds. $B$ : orange color illustrates regions responding to eye or head movements. Green color illustrates regions responding to somatosensory stimulation of the head and neck.

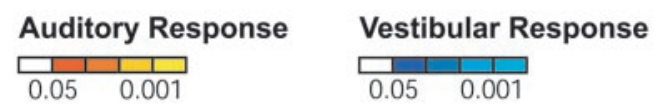

\section{B Eye/Head Movement vs. Neck Somatosensory}
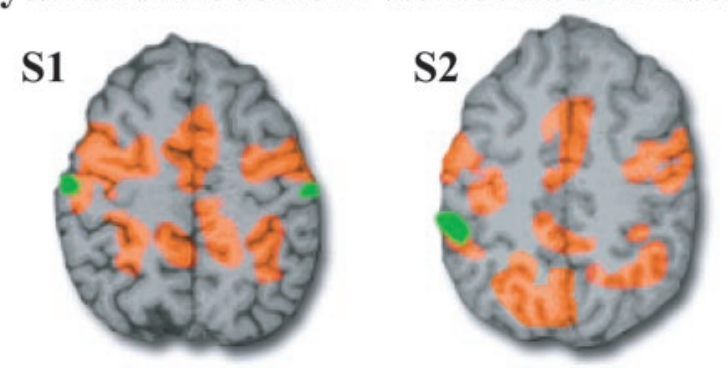

Neck somatosensory
Eye/Head Movement

for oculomotor control. These included PreCS, the medial part of the SEF, the IPS and precuneus, and the LOS. The current findings provide the first functional imaging evidence that such cortical eye fields also subserve visually guided head and gaze movements. These results are consistent with reports from studies of human patients and nonhuman primates on the tight neural linkage between the neural machinery for eye and head movements. Patient AI suffers from congenital abnormalities of the extraocular muscles, resulting in total ophthalmoplegia. However, her visual behavior is relatively unimpaired because she is able to make head-movement saccades that are qualitatively similar to eye-movement saccades in normal subjects, suggesting that common neural mechanisms are responsible for both types of motions (Gilchrist et al. 1998). While cortical areas for oculomotor control were active to a similar degree for eye and head movements in the present experiment, there are important differences between eye and head movements: for example, the head has a large inertia and signals to initiate head motion must be very different from those to guide eye movements. Likewise, feedback signals from either vestibular or stretch receptors are necessary and important in controlling head motion but not eye motion. These neural differences between eye and head movements may occur largely in brain stem nuclei (difficult to image with fMRI); they may exist in cortex but at a smaller spatial scale $(\sim 4 \mathrm{~mm})$ than imaged in the present study; or they may simply be too subtle to observe with fMRI.

In nonhuman primates, stimulation of SEF often produces gaze-shifts involving movements of both the head and the eyes (Sparks et al. 2001). Neurons coding head and gaze position have been described in a number of regions of parietal cortex (reviewed in Colby and Goldberg 1999) including area LIP (Brotchie et al. 1995; Snyder et al. 1998) and area MP (Thier and Andersen 1998), which may be analogous to the IPS and precuneus activations observed in our study and in previous neuroimaging studies of oculomotor control. Neurons in the MT/MST complex respond to eye movements and show responses to vestibular-canal stimulation and optokinetic nystagmus (Bremmer et al. 1997; Ilg and Hoffmann 1993; Thier and Erickson 1992) related to the rate at which the head turns (Andersen et al. 1999). 
A previous study described two distinct foci of activation along the PreCS for overt and covert shifts of visuospatial attention (Beauchamp et al. 2001). The present findings extend these results, with both dorsal and ventral foci showing activity during head movements (as shown in Fig. 3A). The first focus lies in the superior part of the PreCS at the junction with the superior frontal sulcus. The second focus lies along the inferior part of the PreCS at the junction with the inferior frontal sulcus. The superior PreCS focus is thought to contains the human homologue of monkey FEF, known to be responsive to execution of both saccadic and pursuit eye movements (Beauchamp et al. 2001; Berman et al. 1999; Paus 1996; Peralta et al. 1998; Petit and Haxby 1999; Rosano et al. 2001). The more inferior focus has less certain homology, although evidence from nonhuman primates shows several regions of eye-movement producing cortex near the FEF (Fujii et al. 2000; Preuss et al. 1996). In the majority of nonhuman primate studies, the head is held fixed by a head post, meaning that no information can be gathered about the involvement of monkey FEF in head movements. However, recent experiments in monkeys with unrestrained heads show changes in eye, head, and gaze position produced by microstimulation of FEF (Sparks et al. 2001; Tu and Keating 2000), supporting the general contribution of the FEF to coordinated eye and head movements.

Performing eye, head, and gaze movements also leads to robust increases in multiple subcortical areas including basal ganglia, thalamus and SC. Previous PET studies of saccadic eye movements demonstrated a consistent involvement of subcortical structures such as the lenticular nucleus and the thalamus in self-paced (Lang et al. 1994; Law et al. 1998; Petit et al. 1993) and visually and memory-guided saccadic eye movements (Petit et al. 1996). Oculomotor activation of these structures has been more difficult to detect using fMRI, perhaps due to susceptibility effects and less dense vascularization. In the present study, we observed activity in every subject in basal ganglia, thalamus and superior colliculus, perhaps due to the high-field strength ( 3 Tesla) and event-related design used. To date, our findings represent the first demonstration that human superior colliculus is active during eye, head, and gaze movements.

\section{Specific head-and gaze-movement-related activity}

In bilateral perisylvian cortex, responses were observed during head and gaze movement trials but not during eye movements (Figs. 5 and 6). In monkeys, the core vestibular region (primary vestibular cortex) is located in the retroinsular cortex and has been labeled the PIVC. PIVC receives projections from brain stem vestibular nuclei and contains neurons with response properties selective for vestibular stimulation (review in Guldin and Grüsser 1998). Although the homology between monkeys and humans in this region of cortex is especially uncertain, studies of patients (Brandt and Dieterich 1999; Brandt et al. 1994) and functional neuroimaging of invasive vestibular stimulation (reviews in Dieterich and Brandt 2000; Paulesu et al. 1997) suggest that the human homologue of PIVC lies in a perisylvian region including the posterior insula and retroinsular cortex (see Fig. 1 in Bense et al. 2001 for insula anatomy). To better ascertain the location of vestibular cortex in humans, we performed a meta-analysis of neuroim- aging studies that have reported vestibular activation (Bense et al. 2001; Bottini et al. 1994, 2001; Deutschländer et al. 2002; Dieterich et al. 1998; Lobel et al. 1998). As shown in Figs. 6 and 7, these activations consistently lie in the posterior end of the Sylvian fissure, posterior to auditory cortex. The mean location from the meta-analysis was not significantly different from the perisylvian activation observed in the present study. This finding confirms that posterior Sylvian cortex contains core vestibular cortex (the human homologue of PIVC) and provides the first evidence of PIVC activation for vestibular input produced by natural head movements.

Head and gaze movements (but not eye movements) also activated bilateral regions of the PostCS and the paracentral lobule. Right-lateralized activations were observed in the supramarginal gyrus, the superior parietal gyrus. In dorsal occipital cortex, the cuneus showed strong head/gaze-movementrelated activity and a weaker eye-movement response. This region lies near cortex assigned to visual areas V3A and V7, suggesting that these dorsal visual areas (or adjacent areas) are important for coupling visual stimuli with eye, head, and gaze shifts.

Our data also suggest fruitful directions for a understanding of possible homologies between vestibular regions in monkey (review in Guldin and Grüsser 1998) and human cortex (Dieterich and Brandt 2000; Paulesu et al. 1997).

First we consider the large PostCS activation observed near the location of primary somatosensory cortex (SI) that extends from near the central sulcus to the tip of the intraparietal sulcus (Fig. 5). This is consistent with vestibular activation observed in previous studies (Bottini et al. 1994 2001) and may be related to an area in the monkey central sulcus (area $3 \mathrm{aV}$ ) that receives vestibular inputs (Paulesu et al. 1997). A second likely source of this activity is somatosensory feedback from the neck and back of the head during supine head movements, as the neck brushes against the foam pillow. Support for this idea is shown in Fig. 7B, which illustrates overlap between brain areas active during head movements and areas active during tactile stimulation of the back of the head and neck.

A second region of head/gaze movement related activity was observed in the supramarginal gyrus (Brodmann area 40, BA40). This activation is also consistent with that observed during invasive vestibular stimulation (Bense et al. 2001; Bottini et al. 2001; Dieterich and Brandt 2000) and may correspond to the monkey vestibular field located in area $7 \mathrm{~b}$ of inferior parietal cortex (Faugier-Grimaud and Ventre 1989).

\section{Subcortical activity}

Eye and head movements are controlled by a complex network of cortical and subcortical areas. As have all previous fMRI studies of eye movements, the present study examined the cortical network for eye and head movements. However, we saw consistent activity in the regions of the SC. We also saw activity in other brain stem regions, which may include contributions from other nuclei important in the control of eye and head movements, such as the pontine reticular formation, the interstitial nucleus of Cajal, and the cuneiform nucleus. Comprehensive examination of the brain stem eye-movement control nuclei using fMRI requires new developments in three parallel areas: anatomical identification of these structures in vivo based on new advances in pulse sequences (Tuch et al. 
2001); correcting for the high degree of MR physiological noise in these regions caused by motion of the brain stem during heartbeat and respiration; and increases in signal-tonoise ratio to allow detection of BOLD signal change in brain stem structures, perhaps using phased-array coils (de Zwart et al. 2002).

\section{Vestibuloocular reflex}

The vestibuloocular reflex (VOR) serves to keep the visual image still by adjusting the position of the eye in the orbit to compensate for head movements (Carpenter 1988) Brain stem nuclei are the primary coordinators of the VOR, although the cerebellar flocculus serves to modulate the reflex, as do descending projections from cortical eye-movement control areas, both directly and via the SC and the basal ganglia. In the head-movement condition of the present experiment, the VOR would be active, stabilizing the fixation crosshair at the center of gaze despite the head movement toward the target. In the gaze-movement condition, the VOR would be suppressed, as the eyes and head both move toward the target.

\section{Relationship between neuronal responses and the fMRI signal}

While we observed a consistent relationship between eye and head movements and the MR signal from cortical and subcortical brain regions, the exact contributions of different neuronal populations (such as pyramidal motor neurons vs. inhibitory interneurons) to the MR response is unknown. The BOLD fMRI signal is an indirect measure of neuronal activity, reflecting the summed metabolic activity of many neurons filtered by the vasculature. Simultaneous recording of local field potential, multiunit action potentials, and the BOLD fMRI response shows a good correspondence between all three measures (Logothetis et al. 2001) with parametric increases in neuronal firing reflected by concomitant increases in the BOLD signal. Because local field potential shows a slightly better correspondence with the BOLD signal than multiunit activity and because synaptic transmission is thought to more metabolically demanding than action potential production, the BOLD signal may reflect intracortical metabolism and synaptic input, both excitatory and inhibitory (Seidemann et al. 2002), to a given cortical region. fMRI has proven to have remarkable spatial resolution, to the level of individual ocular dominance columns in visual cortex (Cheng et al. 2001). This highly localized response may be mediated via control of individual capillaries in the microvascular bed (Harrison et al. 2002).

We are grateful to Dr. James V. Haxby for many contributions to the research. We also thank K. Lee and S. Marrett for assistance.

\section{REFERENCES}

Andersen RA, Shenoy KV, Snyder LH, Bradley DC, and Crowell JA. The contributions of vestibular signals to the representations of space in the posterior parietal cortex. Ann NY Acad Sci 871: 282-292, 1999.

Anderson TJ, Jenkins IH, Brooks DJ, Hawken MB, Frackowiak RSJ, and Kennard C. Cortical control of saccades and fixation in man: a PET study. Brain 117: 1073-1084, 1994.

Beauchamp MS, Petit L, Ellmore TM, Ingeholm J, and Haxby JV. A parametric fMRI study of overt and covert shifts of visuospatial attention. Neuroimage 14: 310-321, 2001.
Bense S, Stephan T, Yousry TA, Brandt T, and Dieterich M. Multisensory cortical signal increases and decreases during vestibular galvanic stimulation (fMRI). J Neurophysiol 85: 886-899, 2001.

Berman RA, Colby CL, Genovese CR, Voyvodic JT, Luna B, Thulborn KR, and Sweeney JA. Cortical networks subserving pursuit and saccadic eye movements in humans: an fMRI study. Hum Brain Map 8: 209-225, 1999.

Birn RM, Bandettini P, Cox RW, and Shaker R. Event-related fMRI of tasks involving brief motion. Hum Brain Map 7: 106-114, 1999.

Bodis-Wollner I, Bucher SF, Seelos KC, Paulus W, Reiser M, and Oertel WH. Functional MRI mapping of occipital and frontal cortical activity during voluntary and imagined saccades. Neurology 49: 416-420, 1997.

Bottini G, Karnath HO, Vallar G, Sterzi R, Frith CD, Frackowiak RS, and Paulesu E. Cerebral representations for egocentric space: Functional-anatomical evidence from caloric vestibular stimulation and neck vibration. Brain 124: 1182-1196, 2001.

Bottini G, Sterzi R, Paulesu E, Vallar G, Cappa SF, Erminio F, Passingham RE, Frith CD, and Frackowiak RSJ. Identification of the central vestibular projections in man: a positron emission tomography activation study. Exp Brain Res 99: 164-169, 1994.

Brandt $\mathbf{T}$ and Dieterich M. The vestibular cortex. Its locations, functions, and disorders. Ann NY Acad Sci 871: 293-312, 1999.

Brandt T, Dieterich M, and Danek A. Vestibular cortex lesions affect the perception of verticality. Ann Neurol 35: 403-412, 1994.

Bremmer F, Ilg UJ, Thiele A, Distler C, and Hoffmann KP. Eye position effects in monkey cortex. I. Visual and pursuit-related activity in extrastriate areas MT and MST. J Neurophysiol 77: 944-961, 1997.

Brotchie PR, Andersen RA, Snyder LH, and Goodman SJ. Head position signals used by parietal neurons to encode locations of visual stimuli. Nature 375: 232-235, 1995.

Carpenter RHS. Movements of the Eyes, (2nd ed.). London: Pion, 1988.

Cheng K, Waggoner RA, and Tanaka K. Human ocular dominance columns as revealed by high-field functional magnetic resonance imaging. Neuron 32: 359-374, 2001.

Cohen MS. Parametric analysis of fMRI data using linear systems methods. NeuroImage 6: 93-103, 1997.

Colby CL and Goldberg ME. Space and attention in parietal cortex. Annu Rev Neurosci 22: 319-349, 1999.

Corbetta M, Akbudak E, Conturo TE, Drury HA, Linenweber MR, Ollinger JM, Petersen SE, Raichle ME, Snyder AZ, Van Essen DC, and Shulman GL. A common cortical network for attention and eye movements. Neuron 21: 761-768, 1998.

Cox RW. AFNI: software for analysis and visualization of functional magnetic resonance neuroimages. Comput Biomed Res 29: 162-173, 1996.

Cox RW and Hyde JS. Software tools for analysis and visualization of fMRI data. NMR Biomed 10: 171-178, 1997.

Cox RW and Jesmanowicz A. Real-time 3D image registration for functional MRI. Magn Reson Med 42: 1014-1018, 1999.

Darby DG, Nobre AC, Thangaraj V, Edelman R, Mesulam MM, and Warach S. Cortical activation in the human brain during lateral saccades using EPISTAR functional magnetic resonance imaging. Neuroimage 3: 53-62, 1996.

Deutschländer A, Bense S, Stephan T, Schwaiger M, Brandt T, and Dieterich M. Sensory system interactions during simultaneous vestibular and visual stimulation in PET. Hum Brain Mapp 16: 92-103, 2002.

de Zwart JA, Ledden PJ, Kellman P, van Gelderen P, and Duyn JH. Design of a SENSE-optimized high-sensitivity MRI receive coil for brain imaging. Magn Reson Med 47: 1218-1227, 2002.

Dieterich $\mathbf{M}$ and Brandt $\mathbf{T}$. Brain activation studies on visual-vestibular and ocular motor interaction. Curr Opin Neurol 13: 13-18, 2000.

Dieterich M, Bucher SF, Seelos KC, and Brandt T. Horizontal or vertical optokinetic stimulation activates visual motion-sensitive, ocular motor and vestibular cortex areas with right hemispheric dominance — an fMRI study. Brain 121: 1479-1495, 1998.

Dorrichi F, Perani D, Incoccia C, Grassi F, Cappa SF, Bettinardi V, Galati G, Pizzamiglio L, and Fazio F. Neural control of fast-regular saccades and antisaccades: an investigation using positron emission tomography. Exp Brain Res 116: 50-62, 1997.

Faugier-Grimaud S and Ventre J. Anatomic connections of inferior parietal cortex (area 7) with subcortical structures related with vestibulo-ocular function in a monkey (Macaca fascicularis). J Comp Neurol 280: 1-14, 1989. 
Fox PT, Fox JM, Raichle ME, and Burde RM. The role of cerebral cortex in the generation of voluntary saccades: a positron emission tomographic study. J Neurophysiol 54: 348-369, 1985a.

Fox PT, Raichle ME, and Thach WT. Functional mapping of the human cerebellum with positron emission tomography. Proc Natl Acad Sci USA 82: 7462-7466, 1985b.

Fujii N, Mushiake H, and Tanji J. Rostrocaudal Distinction of the Dorsal Premotor Area Based on Oculomotor Involvement. J Neurophysiol 83: 1764-1769, 2000 .

Gilchrist ID, Brown V, Findlay JM, and Clarke MP. Using the eyemovement system to control the head. Proc $R$ Soc Lond B Biol Sci 265: 1831-1836, 1998.

Guldin WO and Grüsser O-J Is there a vestibular cortex? Trends Neurosci 21: 254-259, 1998.

Harrison RV, Harel N, Panesar J, and Mount RJ. Blood capillary distribution correlates with hemodynamic-based functional imaging in cerebral cortex. Cereb Cortex 12: 225-233, 2002.

Heide W, Binkofski F, Seitz RJ, Posse S, Nitschke MF, Freund HJ, and Kompf D. Activation of frontoparietal cortices during memorized triple-step sequences of saccadic eye movements: an fMRI study. Eur J Neurosci 13: 1177-1189, 2001

Ilg UJ and Hoffmann K-P. Functional grouping of the cortico-pretectal projection. J Neurophysiol 70: 867-869, 1993.

Lang W, Petit L, Höllinger P, Pietrzyk U, Tzourio N, Mazoyer B, and Berthoz A. A positron emission tomography study of oculomotor imagery. Neuroreport 5: 921-924, 1994.

Law I, Svarer C, Holm S, and Paulson OB. The activation pattern in normal humans during suppression, imagination and performance of saccadic eye movements. Acta Physiol Scand 161: 419-434, 1997.

Law I, Svarer C, Rostrup E, and Paulson OB. Parieto-occipital cortex activation during self-generated eye movements in the dark. Brain 121: 2189-2200, 1998

Lobel E, Kleine JF, Le Bihan D, Leroy-Willig A, and Berthoz A. Functional MRI of galvanic vestibular stimulation. J Neurophysiol 80: 2699-2709, 1998.

Logothetis NK, Pauls J, Augath M, Trinath T, and Oeltermann A. Neurophysiological investigation of the basis of the fMRI signal. Nature 412: $150-157,2001$

Luna B, Thulborn KR, Strojwas MH, McCurtain BJ, Berman RA, Genovese CR, and Sweeney JA. Dorsal cortical regions subserving visually-guided saccades in humans: an fMRI study. Cereb Cortex 8: 40-47, 1998.

Müri RM, Iba-Zizen MT, Derosier C, Cabanis EA, and Pierrot-Deseilligny C. Location of the human posterior eye field with functional magnetic resonance imaging. J Neurol Neurosurg Psychiatry 60: 445-448, 1996.

Nakashima Y, Momose T, Sano I, Katayama S, Nakajima T, Niwa S-I, and Matsushita M. Cortical control of saccade in normal and schizophrenic subjects: a PET study using a task-evoked rCBF paradigm. Schizophrenia Res 12: 259-264, 1994

Nobre AC, Gitelman DR, Dias EC, and Mesulam MM. Covert visual spatial orienting and saccades: overlapping neural systems. Neuroimage 11: 210 216, 2000.

O'Driscoll GA, Alpert NM, Matthysse SW, Levy DL, Rauch SL, and Holzman PS. Functional neuroanatomy of antisaccade eye movements investigated with positron emission tomography. Proc Natl Acad Sci USA 92: 925-929, 1995.

O'Sullivan EP, Jenkins IH, Henderson L, Kennard C, and Brooks DJ. The functional anatomy of remembered saccades: a PET study. Neuroreport 6 : 2141-2144, 1995.

Paulesu E, Frackowiak RSJ, and Bottini G. Maps of somatosensory systems. In: Human Brain Function, edited by Frackowiak RSJ, Friston KJ, Frith CD, Dolan RJ, and Mazziotta JC. San Diego, CA: Academic, 1997, p. $183-242$.

Paus T. Location and function of the human frontal eye field: a selective review. Neuropsychologia 34: 475-483, 1996.
Paus T, Petrides M, Evans AC, and Meyer E. Role of the human anterior cingulate cortex in the control of oculomotor, manual, and speech responses: a positron emission tomography study. J Neurophysiol 70: 453-469, 1993.

Peralta MRI, Hof PR, and Petit L. Localization and chemoarchitecture of the human frontal eye field. Soc Neurosci Abstr 24: 1147, 1998.

Petit L, Clark VP, Ingeholm JE, and Haxby JV. Dissociation of saccaderelated and pursuit-related activation in human frontal eye fields as revealed by fMRI. J Neurophysiol 77: 3386-3390, 1997.

Petit L, Courtney SM, Ungerleider LG, and Haxby JV. Sustained activity in the medial wall during working memory delays. J Neurosci 18: 94299437, 1998.

Petit L and Haxby JV. Functional anatomy of pursuit eye movements in humans as revealed by fMRI. J Neurophysiol 82: 463-471, 1999.

Petit L, Orssaud C, Tzourio N, Crivello F, Berthoz A, and Mazoyer B. Functional anatomy of a prelearned sequence of horizontal saccades in man. J Neurosci 16: 3714-3726, 1996.

Petit L, Orssaud C, Tzourio N, Salamon G, Mazoyer B, and Berthoz A. PET study of voluntary saccadic eye movements in humans: basal gangliathalamocortical system and cingulate cortex involvement. J Neurophysiol 69: 1009-1017, 1993.

Picard N and Strick PL. Motor areas of the medial wall: a review of their location and functional activation. Cereb Cortex 6: 342-353, 1996.

Preuss TM, Stepniewska I, and Kaas JH. Movement representation in the dorsal and ventral premotor areas of owl monkeys: a microstimulation study [published erratum appears in J Comp Neurol 377: 611, 1997]. J Comp Neurol 371: 649-676, 1996.

Rosano C, Sweeney JA, Melchitzky DS, and Lewis DA. Chemoarchitecture of the human precentral sulcus (PS). Soc Neurosci Abstr 31: 71.28, 2001.

Seidemann E, Arieli A, Grinvald A, and Slovin H. Dynamics of depolarization and hyperpolarization in the frontal cortex and saccade goal. Science 295: 862-865, 2002.

Snyder LH, Grieve KL, Brotchie P, and Andersen RA. Separate body- and world-referenced representations of visual space in parietal cortex. Nature 394: 887-891, 1998.

Sparks DL, Freedman EG, Chen LL, and Gandhi NJ. Cortical and subcortical contributions to coordinated eye and head movements. Vision Res 41: 3295-3305, 2001.

Sweeney JA, Mintun MA, Kwee S, Wiseman MB, Brown DL, Rosenberg DR, and Carl JR. Positron emission tomography study of voluntary saccadic eye movements and spatial working memory. J Neurophysiol 75: 454-468, 1996.

Talairach J and Tournoux P. Co-Planar Stereotaxic Atlas of the Human Brain. New York: Thieme, 1988.

Thier $\mathbf{P}$ and Andersen RA. Electrical microstimulation distinguishes distinct saccade-related areas in the posterior parietal cortex. J Neurophysiol 80 : $1713-1735,1998$

Thier $P$ and Erickson RG. Vestibular input to visual-tracking neurons in area MST of awake rhesus monkeys. Ann NY Acad Sci 656: 960-963, 1992.

Tootell RBH and Taylor JB. Anatomical evidence for MT and additional cortical visual areas in humans. Cereb Cortex 5: 39-55, 1995.

Tu TA and Keating EG. Electrical stimulation of the frontal eye field in a monkey produces combined eye and head movements. J Neurophysiol 84: 1103-1106, 2000.

Tuch DS, Wedeen VJ, Dale AM, George JS, and Belliveau JW. Conductivity tensor mapping of the human brain using diffusion tensor MRI. Proc Natl Acad Sci USA 98: 11697-11701, 2001.

Watson JDG, Myers R, Frackowiak RSJ, Hajnal JV, Woods RP, Mazziotta JC, Shipp S, and Zeki S. Area V5 of the human brain: evidence from a combined study using positron emission tomography and magnetic resonance imaging. Cereb Cortex 3: 79-94, 1993.

Zeki S, Watson JDG, Lueck CJ, Friston KJ, Kennard C, and Frackowiak RSJ. A direct demonstration of functional specialization in human visual cortex. J Neurosci 11: 641-649, 1991 